\title{
Growth Hormone Receptor Antagonist Transgenic Mice Have Increased Subcutaneous Adipose Tissue Mass, Altered Glucose Homeostasis and No Change in White Adipose Tissue Cellular Senescence
}

\author{
Ross Comisford ${ }^{a}$ Ellen R. Lubbers ${ }^{a} \quad$ Lara A. Householder $^{a}$ Ozan Suer $^{a}$ \\ Tamara Tchkoniad James L. Kirkland $^{d}$ Edward O. List ${ }^{a} \quad$ John J. Kopchick ${ }^{a}$ b \\ Darlene E. Berrymana, b, c \\ ${ }^{a}$ Edison Biotechnology Institute, ${ }^{b}$ Department of Biomedical Sciences, Heritage College of Osteopathic Medicine, \\ and 'School of Applied Health Sciences and Wellness, College of Health Sciences and Professions, Ohio University, \\ Athens, Ohio, and d Robert and Arlene Kogod Center on Aging, Mayo Clinic, Rochester, Minn., USA
}

\section{Key Words \\ Growth hormone - Adipose tissue - Cellular senescence . \\ Growth hormone antagonist}

\begin{abstract}
Background: Growth hormone (GH)-resistant/deficient mice experience improved glucose homeostasis and substantially increased lifespan. Recent evidence suggests that long-lived GH-resistant/deficient mice are protected from white adipose tissue (WAT) dysfunction, including WAT cellular senescence, impaired adipogenesis and loss of subcutaneous WAT in old age. This preservation of WAT function has been suggested to be a potential mechanism for the extended lifespan of these mice. Objective: The objective of this study was to examine WAT senescence, WAT distribution and glucose homeostasis in dwarf GH receptor antagonist (GHA) transgenic mice, a unique mouse strain having decreased GH action but normal longevity. Methods: 18-month-old female GHA mice and wild-type (WT) littermate controls were used. Prior to dissection, body composition, fasting blood glucose as well as glucose and insulin tolerance tests were performed. WAT distribution was determined by weighing four distinct WAT depots at the time of
\end{abstract}

dissection. Cellular senescence in four WAT depots was assessed using senescence-associated $\beta$-galactosidase staining to quantify the senescent cell burden, and real-time qPCR to quantify gene expression of senescence markers p16 and IL-6. Results: GHA mice had a 22\% reduction in total body weight, a $33 \%$ reduction in lean mass and a $10 \%$ increase in body fat percentage compared to WT controls. GHA mice had normal fasting blood glucose and improved insulin sensitivity; however, they exhibited impaired glucose tolerance. Moreover, GHA mice displayed enhanced lipid storage in the inguinal subcutaneous WAT depot $(p<0.05)$ and a 1.7-fold increase in extra-/intraperitoneal WAT ratio compared to controls $(p<0.05)$. Measurements of WAT cellular senescence showed no difference between GHA mice and WT controls. Conclusions: Similar to other mice with decreased GH action, female GHA mice display reduced agerelated lipid redistribution and improved insulin sensitivity, but no change in cellular senescence. The similar abundance of WAT senescent cells in GHA and control mice suggests that any protection against generation of senescent cells afforded by decreased GH action, low insulin-like growth factor 1 and/or improved insulin sensitivity in the GHA mice may be offset by their severe adiposity, since obesity is known to increase senescence.

\section{KARGER}

E-Mail karger@karger.com www.karger.com/ger (c) 2015 S. Karger AG, Basel

0304-324X/15/0622-0163\$39.50/0
Darlene E. Berryman

E148 Grover Center, School of Applied Health Sciences and Wellness College of Health Sciences and Professions, Ohio University Athens, OH 45701 (USA)

E-Mail berrymad@ohio.edu 


\section{Introduction}

Aging humans undergo distinctive redistribution of fat characterized by a decrease in subcutaneous adipose tissue (SAT) and an increase in visceral adipose tissue (VAT) [1-3]. SAT is found underneath the skin, mostly in the legs, back and anterior abdominal wall, and is specialized in long-term storage of free fatty acids and triglycerides, while VAT surrounds the abdominal viscera and is more metabolically and lipolytically active than SAT $[3,4]$. Importantly, VAT mass is highly associated with many negative metabolic outcomes seen in obesity, such as impaired glucose and lipid metabolism [3-6]. Thus, age-related lipid redistribution is thought to contribute to the development of aging-associated metabolic diseases including diabetes, cardiovascular disease, metabolic syndrome and cancer [3-6].

Recent studies suggest that age-associated adipose tissue redistribution may be related to cellular senescence of adipocyte progenitor cells $[2,7,8]$. Cellular senescence describes the process in which division-competent cells enter into a state of essentially irreversible growth arrest in response to genomic damage or oncogenic stress [9, 10]. Senescent cells are defined by permanent growth arrest, increased cell size, expression of senescence-associated $\beta$-galactosidase (SA- $\beta$-gal) and activation of tumor suppressor pathways, most commonly the p16 pathway $[9,10]$. In the short term, senescence prevents malignant cells from becoming cancerous; however, senescent cells accumulate with age in a number of tissues, including adipose tissue, with detrimental effects [8-10]. Senescent cells remain metabolically active and secrete a range of chemokines, cytokines, proteases and other factors in what is known as the senescence-associated secretory phenotype [8-11]. These senescence-associated secretory phenotype secretions can disrupt normal cell signaling, promote inflammation and impair tissue function [811]. Moreover, when preadipocytes become senescent, they can no longer differentiate into functional adipocytes; consequently, as senescent cells accrue over time in an adipose tissue depot, the ability to store lipid in that depot becomes compromised $[2,8]$. Thus, accumulation of senescent cells in white adipose tissue (WAT) likely contributes to impaired adipogenesis and decreased lipid storage capacity in subcutaneous WAT depots, resulting in increased lipid being displaced to visceral and ectopic depots over time $[2,7,8]$.

The growth hormone (GH)-induced intracellular signaling pathway is positively correlated with aging. Mice and other species with loss-of-function mutations to the
GH/insulin-like growth factor 1 (IGF-1) axis consistently display delayed aging phenotypes, whereas animals with increases in GH/IGF-1 action experience accelerated aging $[12,13]$. Evidence suggests that this dogma may hold true for humans as well. For example, low IGF-1 levels are predictive of greater longevity in older humans [14], and certain clinical populations with disruptions of the GH/ IGF-1 pathway show similar improvements as mice in terms of glucose homeostasis, cancer and/or longevity [15-18]. Moreover, several single nucleotide polymorphisms in the insulin/IGF-1 pathway have been associated with human longevity [19]. Recently, Stout et al. [7] showed that female mice with decreased GH/IGF-1 activity and increased longevity (GHR-/-, Ames and Snell dwarf mice) experience decreased cellular senescence in WAT, improved capacity for preadipocyte differentiation and reduced age-related lipid redistribution. Additionally, they reported that mice with increased GH signaling experience the opposite effects: increased senescence, impaired adipogenesis and smaller ratio of extra-/intraperitoneal WAT [7]. Their results suggest that chronically elevated GH/IGF-1 action induces senescence of progenitor cells within WAT, either directly or indirectly, leading to impaired adipogenesis, accelerated lipid redistribution and lipotoxicity. Promoting WAT cellular senescence, therefore, could be one mechanism by which the GH/ IGF-1 axis influences the aging process. Moreover, it should be noted that mice with reduced GH action typically exhibit enhanced insulin sensitivity and improved glucose homeostasis $[12,20]$. Since hyperglycemia is associated with increased cellular senescence [21], the decreased senescent cell burden in long-lived GH-resistant/deficient mice could be related to better insulin responsiveness.

$\mathrm{GH}$ receptor antagonist (GHA) mice are unique in that, unlike other GH-resistant/deficient mouse strains, they do not exhibit extended longevity [22]. GHA mice express a GHA which competes with endogenous $\mathrm{GH}$ for binding to the GHR, resulting in reductions in IGF-1 levels to approximately $25 \%$ those of controls and a dwarf phenotype [2224]. GHA mice show important age- and sex-dependent differences with respect to body composition and glucose homeostasis when compared to other dwarf strains of mice, namely GHR gene disrupted (GHR-/-) mice [25, 26]. Therefore, the purpose of the current study was to expand on the report of Stout et al. [7] by examining WAT cellular senescence in GHA mice, a mouse strain with decreased GH/IGF-1 action but no improvement in lifespan. Based on the fact that GHA mice have reduced GH action and that GHA mice have enhanced ability to store fat in subcutaneous depots with age [26], we initially hypothe- 
sized that these mice would exhibit decreased WAT cellular senescence, but not to the same extent as mice with more dramatic reductions in $\mathrm{GH}$ signaling.

\section{Materials and Methods}

\section{Animals}

All animal procedures were approved by the Ohio University Institutional Animal Care and Use Committee. All mice were housed in the facility at the Edison Biotechnology Institute where they were kept on a 14-h light/10-h dark cycle and had ad libitum access to water and normal chow (Prolab RMH 3000, which contains $14 \%$ of energy from fat, $60 \%$ from carbohydrates and $26 \%$ from protein). The development and breeding of GHA transgenic mice has been described previously $[22,24]$. Two cohorts of GHA and wild-type (WT) mice were used in this study. A group of 18-month-old female WT and GHA mice ( $n=6$ for each group) was used for senescence staining and real-time PCR. A separate cohort of 18-month-old female WT and GHA mice (GHA $\mathrm{n}=12$, WT $n=13$ ) was used for all other assays.

\section{Tissue Weights}

All mice were euthanized by $\mathrm{CO}_{2}$ and tissues were immediately excised and weighed. For the senescence studies, five WAT depots were collected, including inguinal subcutaneous (Ing), subscapular (Scap), paraovarian (Para), retroperitoneal (Retro) and mesenteric (Mes). For the mice used for body composition and glucose homeostasis studies, four depots were collected (Ing, Para, Retro, Mes) as well as other select tissues (interscapular brown adipose tissue depot, heart, spleen, kidney, liver and gastrocnemius muscle) for future analyses. All tissues were flash-frozen in liquid nitrogen and stored at $-80^{\circ} \mathrm{C}$. The Ing, Scap and Retro depots are considered extraperitoneal depots while the Para and Mes depots are considered intraperitoneal. Extra-/intraperitoneal WAT ratios were calculated by dividing the sum of the weights of the Ing and Retro depots by the sum of the weights of Para and Mes. Unfortunately, the Scap depot was not included in this calculation because it was not weighed for all mice.

\section{Body Weight and Body Composition}

Body weight and body composition measurements were conducted 1 week prior to dissection. Body composition measurements were done on unanesthetized mice using a Minispec mq benchtop nuclear magnetic resonance analyzer (Bruker Instruments, Billerica, Mass., USA) as previously described [25].

\section{Measurement of Fasting Blood Glucose}

Blood was collected from the tail tip following a 12-h fast. Fasting blood glucose levels were determined using the first drop of blood collected from the tip of the tail. A LifeScan OneTouch glucometer and OneTouch Ultra test strips (LifeScan, Inc., Milpitas, Calif., USA) were used to measure blood glucose.

\section{Glucose and Insulin Tolerance Tests}

Glucose tolerance tests were performed 2 weeks prior to dissection. Mice were fasted for $12 \mathrm{~h}$ prior to the measurements. Intraperitoneal injections of $0.01 \mathrm{ml} 10 \%$ glucose in sterile phosphatebuffered saline per gram body weight were administered. Glucose measurements were performed using a LifeScan OneTouch glucometer and OneTouch Ultra test strips (LifeScan, Inc.) prior to glucose injection and 15, 30, 45, 60, 90, 120 and 150 min after injection. Insulin tolerance tests were performed 1 week prior to dissection. Intraperitoneal injections of $0.01 \mathrm{ml}$ of $0.075 \mathrm{U} / \mathrm{ml}$ insulin (Humulin ${ }^{\circledR}$; Eli Lilly and Company) per gram body weight were administered. Blood glucose measurements using a LifeScan OneTouch glucometer and test strips (LifeScan, Inc.) were performed before insulin injection and 15, 30, 45, 60, 90 and 120 min after injection. Mice were not fasted prior to insulin tolerance tests but were denied access to food during the tests.

\section{Senescence-Associated $\beta$-Galactosidase Staining}

To determine the accumulation of senescent cells, dissected adipose tissue was stained for SA- $\beta$-gal activity immediately post dissection as described previously [7]. Briefly, adipose tissue was fixed for $10 \mathrm{~min}$ in $10 \%$ formaldehyde with $1 \%$ glutaraldehyde, incubated overnight at $37^{\circ} \mathrm{C}$ in a staining solution containing $\mathrm{X}$ galactose $(1 \mathrm{mg} / \mathrm{ml} \mathrm{X}$-gal, $40 \mathrm{~mm}$ citric acid/Na phosphate buffer, $\mathrm{pH} 6.0$ ), then rinsed and stored in phosphate-buffered saline at $4^{\circ} \mathrm{C}$. Percentages of SA- $\beta$-gal-positive cells were determined by comparing phase and DAPI images of four different fields of each sample.

\section{Real-Time PCR}

Adipose tissue from 18-month-old female GHA and WT mice was flash-frozen at the time of dissection and stored at $-80^{\circ} \mathrm{C}$. RNA extraction was performed using TRIzol reagent following the manufacturer's protocol (Fisher Scientific). cDNA was synthesized using Maxima First Strand cDNA Synthesis Kits and quantitative real-time PCR was performed using Maxima SYBR Green/Fluorescein qPCR Master Mix (Thermo Scientific) with a Bio-Rad iCycler Thermal Cycler (Bio-Rad Laboratories, Inc., Hercules, Calif., USA). The primer sequences used for IL-6 were $3^{\prime}$-TCC GGC ACC AAC AGT GGT CG-5' forward and $3^{\prime}$-CCT TTA CTC TTT TCT CAA CAC GT- $5^{\prime}$ reverse, and for p16 primers $3^{\prime}$-CGC TCT GGC TTT CGT GAA C-5' forward and $3^{\prime}$-TTG CCC ATC ATC ATC ACC TGG-5' reverse. Expression levels were normalized to the housekeeping genes beta 2 microglobulin $(B 2 \mathrm{~m})$ and ribosomal protein S3 (Rps3). For Rps3 the primer sequences were $3^{\prime}$-ATC AGA GAG TTG ACC GCA GTT-5' forward and $3^{\prime}$-AAT GAA CCG AAG CAC ACC ATA $-5^{\prime}$ reverse. For B2m the primer sequences were $3^{\prime}$ CTG GTC TTT CTA TAT CCT GGC T-5' forward and $3^{\prime}$-CAT GTC TCG ATC CCA GTA GAC-5' reverse. Analysis of qPCR data was performed with the Biogazelle qbasePLUS software.

\section{Statistical Analyses}

Comparisons were made either using Student's unpaired t test or, where multiple comparisons were made, by two-way ANOVA followed by Fisher's LSD post hoc tests. All statistical analyses were performed using SPSS version 17.0.

\section{Results}

\section{Body Length, Body Composition, Depot Weights and} Extra-/Intraperitoneal WAT Ratio

As expected, 18-month-old female GHA mice had significantly shorter body length (GHA $87.3 \pm 0.6 \mathrm{~cm}$ vs. WT
WAT Senescence and Glucose

Homeostasis in GHA Mice 
Fig. 1. Body composition and WAT distribution in 18-month-old female GHA ( $\mathrm{n}=$ $12)$ and WT $(n=13)$ mice. a Comparison of absolute body weight. b Fat, lean and fluid percentage was determined by dividing fat, lean and fluid mass by total body weight. c Relative WAT depot weight for the Ing, Para, Retro and Mes depots was determined by dividing absolute depot weights by total body weight for each mouse. $\mathbf{d}$ The ratio of extra- to intraperitoneal WAT mass was determined by dividing the combined mass of the Ing and Retro depots by the combined mass of the Para and Mes depots. Data are expressed as mean \pm SEM. * Significantly different from WT littermate controls $(\mathrm{p}<0.05)$.

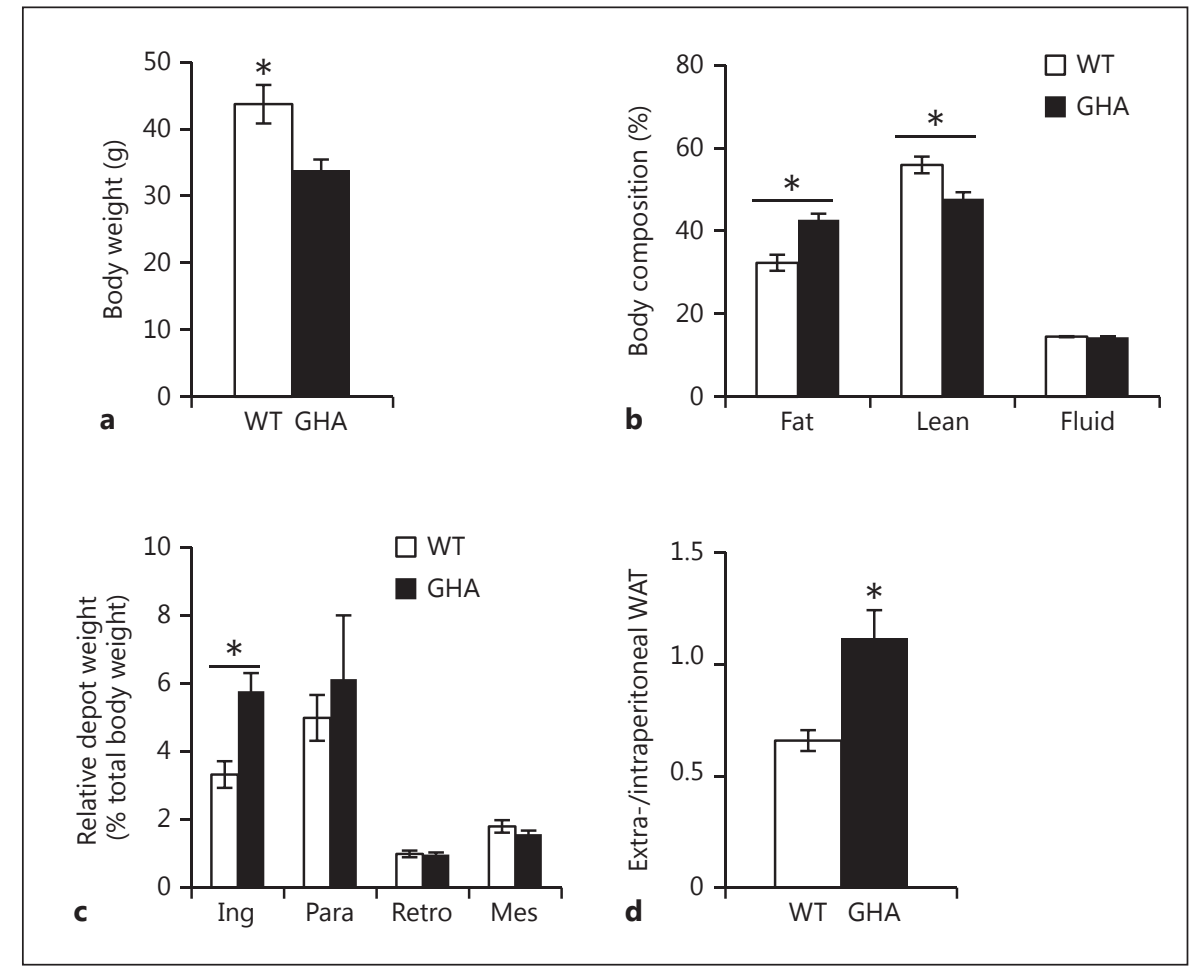

$100.6 \pm 0.7 \mathrm{~cm}, \mathrm{p}<0.001)$ and significantly lower body weight than littermate controls (GHA $33.8 \pm 1.7 \mathrm{~g}$ vs. WT $43.7 \pm 2.9$ g, p $<0.01$, fig. 1a). Thus, the GHA mice in this study can be considered dwarf compared to the WT mice. In terms of body composition, GHA mice had significantly decreased lean mass (GHA $15.9 \pm 0.5 \mathrm{~g}$ vs. WT $23.8 \pm 0.8 \mathrm{~g}, \mathrm{p}<0.001)$ and significantly decreased fluid mass (GHA $4.9 \pm 0.2 \mathrm{~g}$ vs. WT $6.3 \pm 0.4 \mathrm{~g}, \mathrm{p}<0.01$ ). However, there was no significant difference in absolute fat mass compared to WT mice (GHA $14.6 \pm 1.1 \mathrm{~g}$ vs. WT $14.8 \pm 1.8 \mathrm{~g}$ ). Since GHA mice had significantly decreased total body weight, we also assessed fat, lean, and fluid composition as a percentage of total body weight. When normalized to total body weight, GHA mice exhibited significantly increased fat percentage $(\mathrm{p}<0.001$, fig. $1 \mathrm{~b})$, significantly decreased lean percentage $(\mathrm{p}<0.01$, fig. $1 \mathrm{~b})$ and no difference in fluid percentage (fig. 1b). Given the well-known influence of fat depot localization on the tissues' metabolic and endocrine functions, we assessed weights of four distinct white adipose depots: Ing, Retro, Para and Mes. The Ing depot surrounds the inguinal lymph node and is classified as subcutaneous and extraperitoneal because it lies underneath the skin and outside the peritoneal cavity. The Retro depot is found behind the kidneys outside the peritoneum and is therefore classified as extraperitoneal but not subcutaneous. The Para depot, which surrounds the ovaries, is considered intraperitoneal. It is commonly referred to as a visceral depot; however, it does not fit the strict definition of VAT as it does not drain into the portal vein. The Mes depot, which is found intertwined along the length of the intestines, is considered intraperitoneal and is the only true visceral WAT depot in mice. No significant differences were found between genotypes with respect to the absolute mass of any of the individual adipose tissue depots examined. However, after normalizing to total body weight, GHA mice did show a significant increase in the relative mass of the Ing depot ( $p<0.05$, fig. 1c). To further assess the adipose distribution, the ratio of extra- to intraperitoneal WAT was calculated for GHA and control mice. This metric revealed a 1.7 -fold increase in the extra-/intraperitoneal WAT ratio in GHA mice versus WT littermates ( $p<0.01$, fig. $1 d)$.

\section{Glucose Homeostasis}

There was no significant difference in fasting blood glucose (fig. 2a) or fasting insulin levels (fig. 2b) between GHA mice and WT littermate controls. Interestingly, GHA mice displayed enhanced insulin sensitivity. During insulin tolerance tests GHA mice showed significant- 
Fig. 2. Glucose homeostasis in 18-monthold female GHA $(\mathrm{n}=12)$ and WT $(\mathrm{n}=13)$ mice. a Comparison of plasma glucose levels after a 12-h fast. b Comparison of circulating insulin levels after a 12 -h fast. c Insulin tolerance tests showing glucose levels following intraperitoneal injection of 0.075 $\mathrm{U} / \mathrm{ml}$ insulin. d Glucose tolerance tests showing the change in glucose levels following intraperitoneal injection of $10 \%$ glucose solution at $0.01 \mathrm{ml} / \mathrm{g}$ body weight. e Comparison of glucose tolerance test area under the curve (AUC) after 1 and $2 \mathrm{~h}$. Data are expressed as mean \pm SEM. * Significantly different from WT littermate controls $(\mathrm{p}<0.05)$.
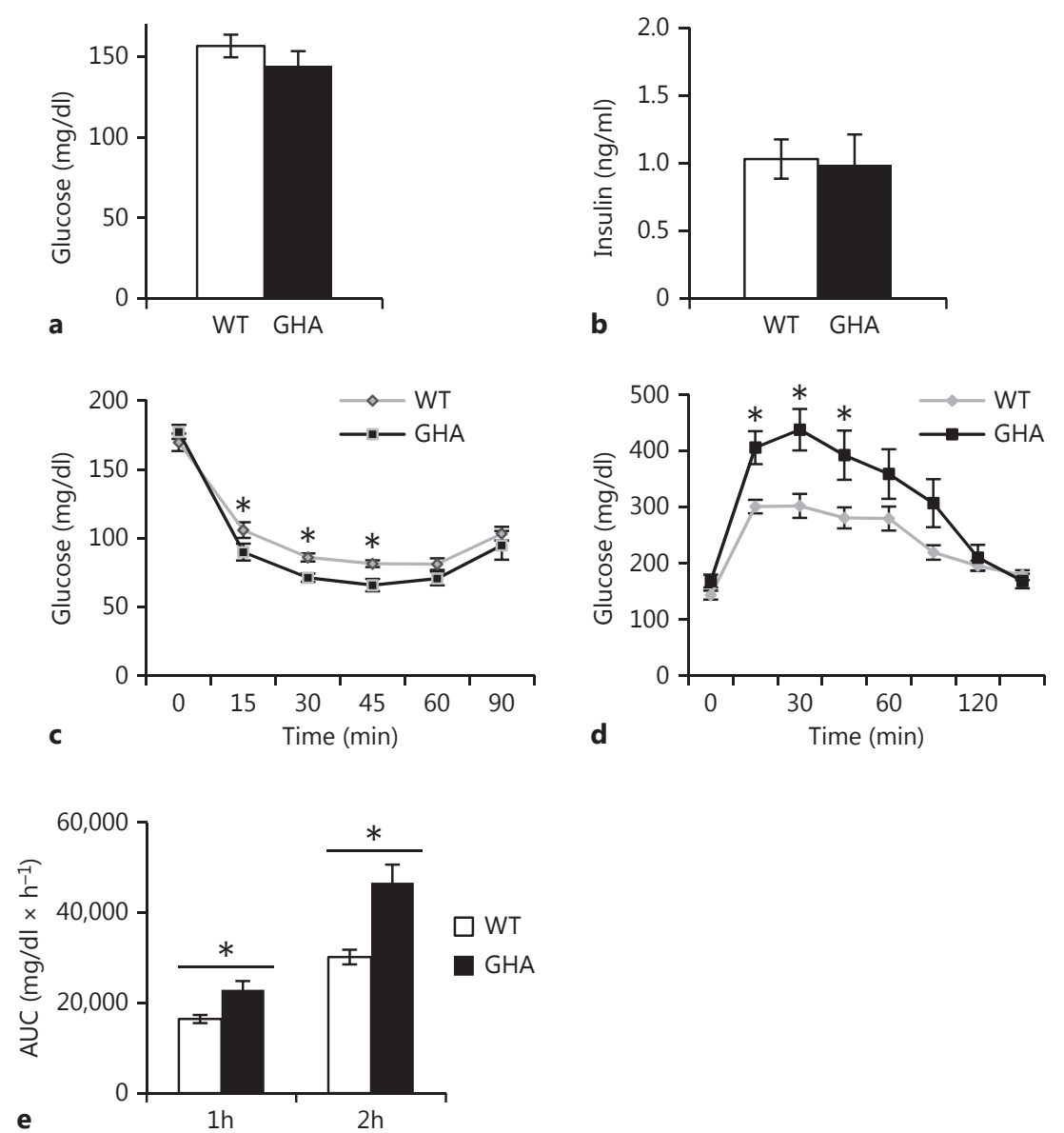

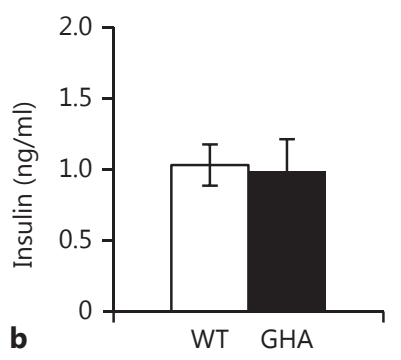


Fig. 3. WAT senescence results for 18month-old female GHA $(\mathrm{n}=6)$ and WT $(n=6)$ mice. a Representative images of Ing WAT from WT (top row) and GHA (bottom row) mice. Images on the left show nuclei stained with DAPI viewed under fluorescence settings (nuclei are light blue); images on the right show the same field viewed under phase contrast settings to visualize SA- $\beta$-gal-positive senescent cells. SA- $\beta$-gal-positive senescent cells give off a blue color when stained with X-galactose (indicated by arrows). The percentage of SA- $\beta$-gal-positive cells is calculated by dividing the number of SA- $\beta$-gal-positive cells by the number of nuclei in each image and the results represent the average of four images taken for each depot from each mouse. b Percentage of SA- $\beta$-gal-positive cells in the Ing, Scap, Para, Retro and Mes WAT depots in 18-month-old female GHA $(\mathrm{n}=6)$ and WT $(\mathrm{n}=6)$ mice. Superscript letters refer to the mean of the depot. Means showing a common superscript letter are not significantly different $(\mathrm{p}>0.05)$. Data are expressed as mean \pm SEM.
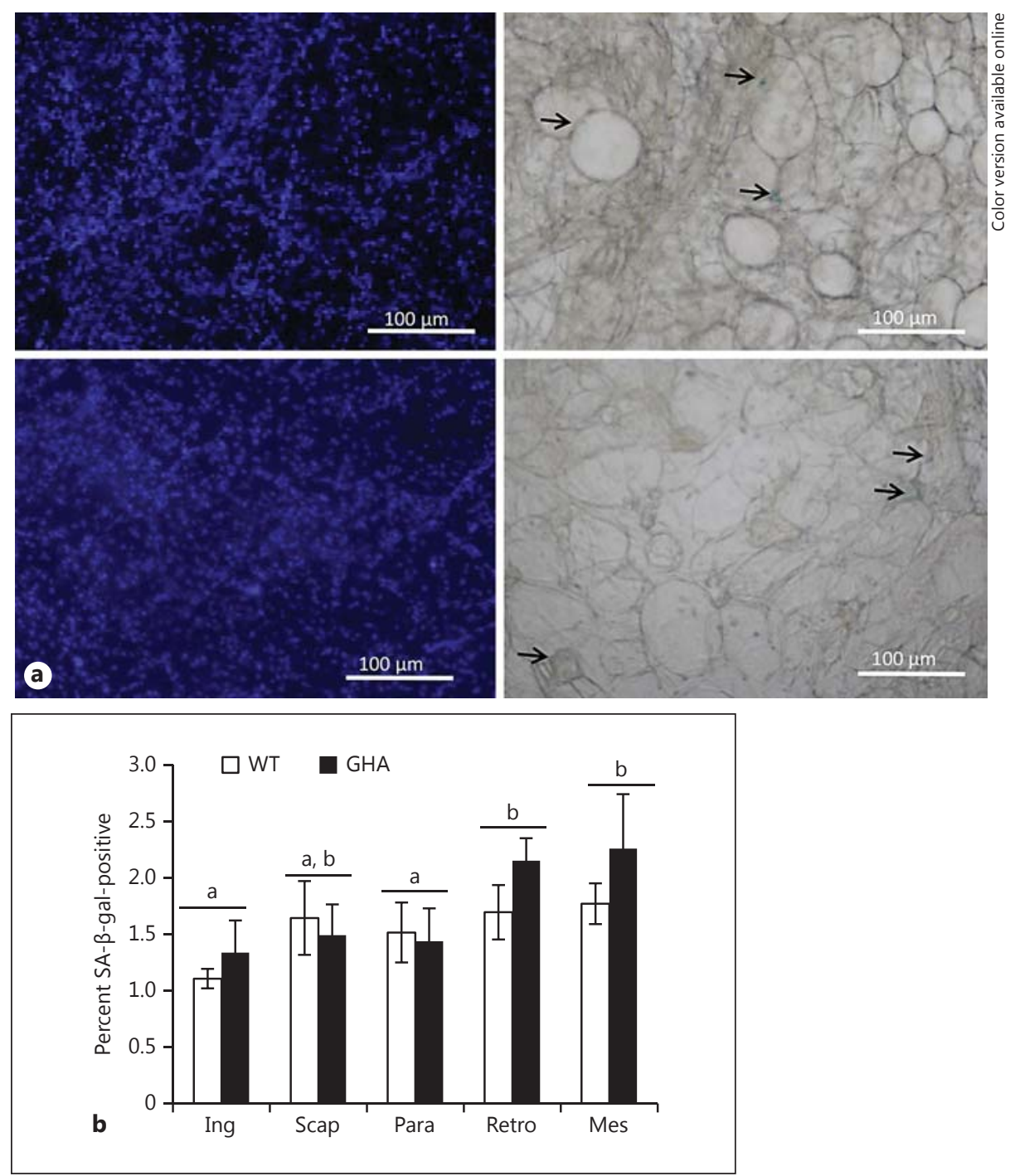

Thus, discovering the physiological differences between GHA mice and long-lived GH-resistant/deficient mice could help pinpoint traits that are important for extending lifespan. Previous research has suggested that differences in adipose tissue mass and distribution between GHA and long-lived GHR-/- mice could be important factors contributing to differences in lifespan and metabolism between these two lines $[25,26]$. WAT cellular senescence has been implicated as a contributing factor to adipose tissue redistribution and impairment of adipogenesis during the aging process $[2,7,8]$. Indeed, 18-month-old female GHR-/- mice are reported to have decreased WAT senescence, which is thought to contribute to their marked expansion of subcutaneous WAT [7]. The current study is the first to examine WAT cellular senescence in GHA mice. Our results indicate that, at 18 months of age, female GHA mice experience enhanced lipid storage in extraperitoneal depots in conjunction with normal glucose and insulin levels, improved insulin sensitivity and impaired glucose tolerance; however, we did not detect any difference in WAT cellular senescence in GHA mice compared to littermate controls.

The majority of previous studies examining the impact of the GHA genotype on body size and composition have focused on male mice. These studies show that male GHA mice have reduced body weight at young ages, but by approximately 11 months of age they no longer differ from controls in total body weight due to extraordinary gains in fat mass $[22,25,26]$. Fewer studies have been published 

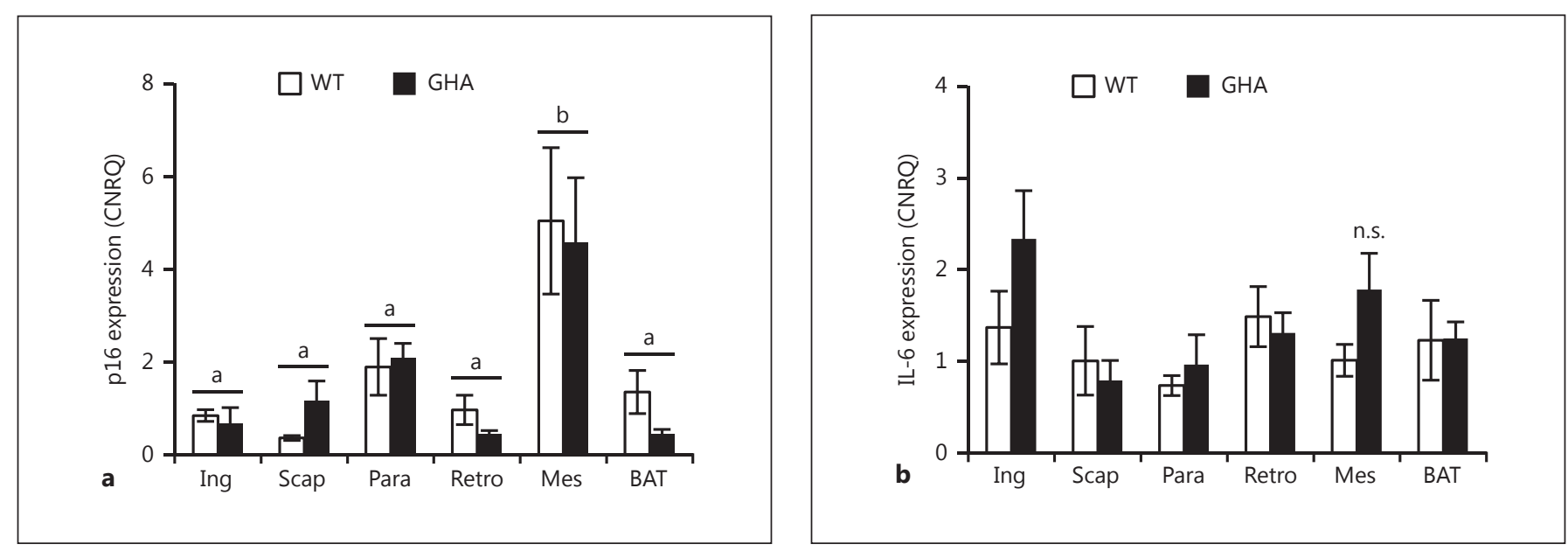

Fig. 4. Gene expression of senescent markers in 18 -month-old female GHA $(n=6)$ and WT $(n=6)$ mice. a Comparison of p16 expression. b Comparison of IL-6 expression. Data are expressed as mean \pm SEM. Superscript letter refer to the mean of the depot. Means showing a common superscript letter are not significantly different $(\mathrm{p}>0.05)$. BAT $=$ Interscapular brown adipose tissue; $n . s .=$ no significant difference.

examining female GHA mice. A prior study that longitudinally assessed body weight changes in female GHA mice revealed that, unlike male GHA mice, females retain significantly lower body weights than littermate controls at least until 84 weeks of age [26]. Additionally, the GHA mice in this study had decreased body length, which is consistent with previous reports that GHA mice are dwarf compared to WT mice $[22,24]$. In terms of body composition, male and female GHA mice have been shown to have increased body fat percentage and decreased lean fat percentage throughout life, although males show greater increases in adiposity as they age than do females $[22,26]$. Our data of 18-month-old female GHA mice showed a $22 \%$ reduction in total body weight, a $33 \%$ reduction in lean mass and a $10 \%$ increase in body fat percentage, consistent with those previous reports.

Increased adiposity in GH-resistant/deficient mice is associated with preferential expansion of SAT [7, 26, 27]. This feature has been suggested to be important for the improved metabolic health seen in these mice as SAT is thought to play a metabolically protective role by sequestering cytotoxic fatty acids for long-term energy storage $[2,4]$. Studies with GHA mice indicate that they too display enhanced WAT storage in subcutaneous depots. For example, 5-month-old male and female GHA mice show significantly increased subcutaneous fat on both a lowand a high-fat diet in both sexes [28], and expanded SAT mass was also reported in 20-month-old female GHA mice [26]. In agreement with these previous studies, we report a significant relative enlargement of the Ing depot in 18-month-old female GHA mice. Moreover, we observed an approximate 1.7-fold increase in the ratio of extra- to intraperitoneal WAT in GHA mice compared to WT controls. This increase is not as dramatic as that reported for 18-month-old female mice from the long-lived GH-resistant/deficient mouse strains (Ames, Snell and GHR-/- mice exhibit increases in the extra-/intraperitoneal WAT ratio of 3-, 2- and 2.3-fold, respectively) [7]; however, a direct comparison is not possible since Stout et al. [7] included one additional extraperitoneal depot (the Scap depot) in their calculation. Previous reports support the notion that GHA mice have reduced lipid redistribution compared to WT mice, although evidence suggests that GHA mice have a more generalized fat distribution than do GHR-/- mice. For example, we previously found that both GHA and GHR-/- mice gain substantial fat mass in the Ing depot as they age, but GHA mice gain significantly more total fat mass than GHR-/mice [26]. Additionally, we show that with advancing age, GHA mice gain greater fat mass in non-subcutaneous depots than GHR-/- mice [26]. The greater overall adipose load in GHA mice may be offsetting the protective benefits of enhanced subcutaneous fat storage that are seen in long-lived GH-resistant/deficient mutants, such as improved glucose homeostasis and enhanced lifespan. Nonetheless, enhanced lipid storage in subcutaneous depots in GHA mice could help explain why these mice are protected from metabolic impairment that would be an- 
ticipated from the extreme obesity they develop in old age or in the context of high-fat diet feeding $[26,28]$.

Long-lived mice with reductions in the GH/IGF-1 axis generally display improvements in glucose homeostasis, and this is believed to be an important factor in the extended lifespan of these animals [12, 20]. Long-lived GHR-/- mice, for instance, remain hypoinsulinemic and insulin-sensitive throughout life [22]. Male GHA mice have low to normal glucose and insulin levels in early life but become hyperinsulinemic by approximately 16 months of age $[22,23,26,28]$. Few studies have longitudinally examined glucose homeostasis in female GHA mice. We recently showed that female GHA mice have decreased blood glucose at younger ages followed by normoglycemia from 52 weeks onward [26]. Additionally, we previously found that female GHA mice have normal circulating insulin levels up to 84 weeks of age and normal glucose tolerance at 73 weeks ( $\sim 16$ months) of age [26]. Similar to previous reports, we report here that, at 18 months of age, female GHA mice have normal fasting blood glucose and insulin levels. However, we found that GHA mice have enhanced insulin sensitivity and a significant decrease in glucose tolerance compared with controls, which has not been previously reported. This paradoxical result could potentially be explained by a decreased capacity for insulin secretion from the pancreas, likely due to decreased islet cell mass, as is suggested for GHR-/- mice [29]. Indeed, a study by Guo et al. [29] showed that restoring pancreatic IGF-1 levels to normal in GHR-/- mice results in increased islet cell mass, improved insulin secretion and improved glucose tolerance. Future studies are needed to more thoroughly assess the changes in glucose homeostasis that are occurring in GHA mice of both sexes, particularly at older age points.

The accumulation of senescent cells in subcutaneous WAT is thought to impair adipogenesis in this depot, leading to increased lipid storage in visceral and ectopic sites $[2,8]$. Of note, the SA- $\beta$-gal staining assay used in this study is not able to distinguish among different WAT cell types. The senescence positivity could arise from any division-competent cells in WAT, including preadipocytes, endothelial progenitor cells, immune progenitor cells or other precursor cells that may be present in WAT. Senescence of preadipocytes directly limits the capacity for adipogenesis while senescence of non-adipose cells could impair adipogenesis indirectly by promoting inflammation [8]. Stout et al. [7] previously showed that long-lived Snell and GHR-/- dwarf mice have increased extraperitoneal WAT storage as well as reduced WAT senescent cell accumulation. Thus, the finding of preserva- tion of extraperitoneal WAT in GHA mice predicts that these mice would show decreased cellular senescence in extraperitoneal WAT depots. Our results, however, did not reveal any significant differences in WAT senescence between GHA and WT mice by SA- $\beta$-gal staining or by gene expression analysis of the senescence markers p16 and IL-6. Comparable senescence prevalence between GHA and WT in the current study suggests that the conservation of subcutaneous WAT mass in old GHA mice is not the result of decreased cellular senescence. However, based on evidence showing that obesity increases cellular senescence [30, 31], one could argue that GHA mice would be expected to show increased WAT cellular senescence compared to WT mice due to their increased adiposity. Thus, it is conceivable that the dampened GH/ IGF-1 signaling in these mice protects against an obesitydriven increase in WAT cellular senescence.

Why might GHA mice differ from other $\mathrm{GH}$-resistant/ deficient mice with regard to WAT cellular senescence? Long-lived Ames, Snell and GHR-/- mice have reduced WAT senescence, and all of these mouse strains exhibit reduced IGF-1, glucose and/or insulin levels throughout their lifespan $[32,33]$, all of which are known to promote senescence in vitro [34-36]. Conversely, short-lived GH transgenic mice display increased WAT senescence as well as increased IGF-1, glucose and insulin levels [7, 33]. GHA mice show reduced IGF-1 levels, but not to the same extent as the long-lived GH-resistant/deficient mice [22, 23 ]. Thus, it could be that the reduction in IGF-1 experienced by GHA mice is not sufficient to elicit benefits. Alternatively, normal levels of WAT senescence in GHA mice could be due to lack of improvements in glucose homeostasis in GHA mice compared to the long-lived mouse strains. Indeed, elevated glucose [34] and insulin [36] can drive cellular senescence in cell culture experiments; moreover, diabetes is associated with increased senescence in multiple tissues in humans and experimental animals [21]. Long-lived GHR-/- mice show low to normal glucose levels, very low circulating insulin levels and substantially enhanced insulin sensitivity throughout their lifespan [32]. Likewise, both Ames and Snell dwarf mice exhibit severely reduced circulating levels of plasma insulin and glucose [33]. GHA mice, on the other hand, do not show consistent improvements in glucose or insulin throughout their lifespan, with some impairment with advancing age $[22,26]$. However, it should be noted that GHA mice do not have increased senescence despite obesity, suggesting that decreased IGF-1 and/or normal glucose homeostasis in these mice might afford some protection against obesity-induced cellular senescence. It is im- 
portant to note that GHA mice remain a relatively understudied mouse strain in comparison to those mouse lines with lifespan extension. Thus, there are numerous other physiological mechanisms related to aging which remain unexplored in the GHA mouse strain that could potentially explain their apparent lack of life extension. GHR-/- mice, for instance, are reported to exhibit delayed reproductive capacity [37], elevated antioxidant enzyme levels [38], increased energy expenditure per unit body weight [39], reduced core body temperature [40], reduced thyroid hormone levels [40] and increased corticosteroid levels [40]. All these factors have been implicated as being potentially important to the extended lifespan of GHR-/- mice, but have yet to be examined in GHA mice. Thus, there are numerous avenues for future studies using these animals that will be helpful in determining the factors that are most influential to lifespan extension.

In summary, this study assessed the impact of the GHA genotype on cellular senescence in WAT of older female mice. Here, we show that 18-month-old female GHA mice have enhanced lipid storage in extraperitoneal adipose tissue. Also, GHA mice exhibit euglycemia and insulin sensitivity despite having increased adiposity. These results support the notion that improved ability to sequester fatty acids in subcutaneous WAT depots is important for the 'healthy' obese phenotype of GHA mice. Contrary to what was previously reported for the longlived GH-resistant/deficient dwarf mice, the preservation of subcutaneous WAT in female GHA mice was not as- sociated with any change in WAT senescent cell burden in the present study. This finding suggests that reduced WAT senescence is not required for the enhanced subcutaneous WAT storage seen in GHA mice. Moreover, our findings are consistent with the intriguing possibility that senescent cell burden is related to lifespan and/or health span. That is, senescent cell abundance is low and lifespan increased in Ames, Snell and GHR-/- mice [7], while we report here that senescent cell burden is similar to controls in GHA mice, which have normal lifespan. Additionally, normal levels of senescence in GHA WAT in the context of increased adiposity could potentially be interpreted as a positive outcome, given that obesity is known to accelerate WAT senescence $[30,31]$.

\section{Acknowledgements}

This work was supported by the State of Ohio's Eminent Scholar Program that includes a gift from Milton and Lawrence Goll (J.J.K.), National Institutes of Health grant AG031736 (J.J.K., D.E.B., E.O.L.), the Diabetes Institute at Ohio University (D.E.B., R.C., L.A.H., E.R.L.), and the American Veterans (J.J.K., E.R.L.). These funding sources had no involvement in the study design, the data collection, the data interpretation, the writing of the report or the decision to publish this work.

\section{Disclosure Statement}

The authors report no conflicts of interest.

\section{References}

$\checkmark 1$ Kuk JL, et al: Age-related changes in total and regional fat distribution. Ageing Res Rev 2009;8:339-348.

-2 Tchkonia T, et al: Mechanisms and metabolic implications of regional differences among fat depots. Cell Metab 2013;17:644-656.

13 Wajchenberg BL: Subcutaneous and visceral adipose tissue: their relation to the metabolic syndrome. Endocr Rev 2000;21:697-738.

4 Ibrahim MM: Subcutaneous and visceral adipose tissue: structural and functional differences. Obes Rev 2010;11:11-18.

5 Goodpaster BH, et al: Association between regional adipose tissue distribution and both type 2 diabetes and impaired glucose tolerance in elderly men and women. Diabetes Care 2003;26:372-379.

6 Shuster A, et al: The clinical importance of visceral adiposity: a critical review of methods for visceral adipose tissue analysis. Br J Radiol 2012;85:1-10.

WAT Senescence and Glucose

Homeostasis in GHA Mice
7 Stout MB, et al: Growth hormone action predicts age-related white adipose tissue dysfunction and senescent cell burden in mice. Aging (Albany NY) 2014;6:575-586.

8 Tchkonia T, et al: Fat tissue, aging, and cellular senescence. Aging Cell 2010;9:667-684.

-9 Rodier F, Campisi J: Four faces of cellular senescence. J Cell Biol 2011;192:547-556.

10 Jeyapalan JC, Sedivy JM: Cellular senescence and organismal aging. Mech Ageing Dev 2008; 129:467-474.

11 Newsholme P, de Bittencourt PI Jr: The fat cell senescence hypothesis: a mechanism responsible for abrogating the resolution of inflammation in chronic disease. Curr Opin Clin Nutr Metab Care 2014;17:295-305.

12 Bartke A, et al: Links between growth hormone and aging. Endokrynol Pol 2013;64:46-52.

13 Junnila RK, et al: The GH/IGF-1 axis in ageing and longevity. Nat Rev Endocrinol 2013; 9:366-376.
14 Milman S, et al: Low insulin-like growth factor-1 level predicts survival in humans with exceptional longevity. Aging Cell 2014;13: 769-771.

15 Laron Z: Laron syndrome (primary growth hormone resistance or insensitivity): the personal experience 1958-2003. J Clin Endocrinol Metab 2004;89:1031-1044.

16 Guevara-Aguirre J, et al: Growth hormone receptor deficiency is associated with a major reduction in pro-aging signaling, cancer, and diabetes in humans. Sci Transl Med 2011;3: 70 ra13.

17 Steuerman R, Shevah O, Laron Z: Congenital IGF1 deficiency tends to confer protection against post-natal development of malignancies. Eur J Endocrinol 2011;164:485-489.

18 Suh Y, et al: Functionally significant insulinlike growth factor I receptor mutations in centenarians. Proc Natl Acad Sci USA 2008; 105:3438-3442. 
19 Pawlikowska L, et al: Association of common genetic variation in the insulin/IGF1 signaling pathway with human longevity. Aging Cell 2009;8:460-472.

20 Bartke A: Impact of reduced insulin-like growth factor-1/insulin signaling on aging in mammals: novel findings. Aging Cell 2008;7: 285-290.

-21 Palmer AK, Tchkonia T, LeBrasseur NK, Chini EN, Xu M, Kirkland JL: Cellular senescence in type 2 diabetes: a therapeutic opportunity. Diabetes 2015;64:2289-2298.

22 Coschigano KT, et al: Deletion, but not antagonism, of the mouse growth hormone receptor results in severely decreased body weights, insulin, and insulin-like growth factor I levels and increased life span. Endocrinology 2003;144:3799-3810.

23 Yakar S, et al: Inhibition of growth hormone action improves insulin sensitivity in liver IGF-1-deficient mice. J Clin Invest 2004;113. 96-105.

24 Chen WY, et al: Functional antagonism between endogenous mouse growth hormone $(\mathrm{GH})$ and a GH analog results in dwarf transgenic mice. Endocrinology 1991;129:14021408.

25 Berryman DE, et al: Comparing adiposity profiles in three mouse models with altered GH signaling. Growth Horm IGF Res 2004 $14: 309-318$
26 Berryman DE, et al: A dwarf mouse model with decreased GH/IGF-1 activity that does not experience life-span extension: potential impact of increased adiposity, leptin, and insulin with advancing age. J Gerontol A Biol Sci Med Sci 2014;69:131-141.

27 Berryman DE, et al: Growth hormone and adipose tissue: beyond the adipocyte. Growth Horm IGF Res 2011;21:113-123.

28 Yang T, et al: Growth hormone receptor antagonist transgenic mice are protected from hyperinsulinemia and glucose intolerance despite obesity when placed on a HF diet. Endocrinology 2015;156:555-564.

29 Guo Y, et al: Pancreatic islet-specific expression of an insulin-like growth factor-I transgene compensates islet cell growth in growth hormone receptor gene-deficient mice. Endocrinology 2005; 146:2602-2609.

30 Minamino T, et al: A crucial role for adipose tissue p53 in the regulation of insulin resistance. Nat Med 2009;15:1082-1087.

31 Villaret A, et al: Adipose tissue endothelial cells from obese human subjects: differences among depots in angiogenic, metabolic, and inflammatory gene expression and cellular senescence. Diabetes 2010;59:2755-2763.

32 List EO, et al: Endocrine parameters and phenotypes of the growth hormone receptor gene disrupted (GHR-/-) mouse. Endocr Rev 2011;32:356-386.

33 Bartke A, et al: Does growth hormone prevent or accelerate aging? Exp Gerontol 1998;33: 675-687.
34 Blazer S, et al: High glucose-induced replicative senescence: point of no return and effect of telomerase. Biochem Biophys Res Commun 2002;296:93-101.

35 Handayaningsih AE, et al: IGF-I enhances cellular senescence via the reactive oxygen species-p53 pathway. Biochem Biophys Res Commun 2012;425:478-484.

36 Matsui-Hirai H, et al: Dose-dependent modulatory effects of insulin on glucose-induced endothelial senescence in vitro and in vivo: a relationship between telomeres and nitric oxide. J Pharmacol Exp Ther 2011;337:591-599.

37 Chandrashekar V, et al: Pituitary and testicular function in growth hormone receptor gene knockout mice. Endocrinology 1999; 140: 1082-1088.

38 Hauck SJ, et al: Antioxidant enzymes, freeradical damage, and response to paraquat in liver and kidney of long-living growth hormone receptor/binding protein gene-disrupted mice. Horm Metab Res 2002;34:481-486.

39 Longo KA, et al: Daily energy balance in growth hormone receptor/binding protein (GHR-/-) gene-disrupted mice is achieved through an increase in dark-phase energy efficiency. Growth Horm IGF Res 2010;20:7379.

40 Hauck SJ, et al: Reduced levels of thyroid hormones, insulin, and glucose, and lower body core temperature in the growth hormone receptor/binding protein knockout mouse. Exp Biol Med (Maywood) 2001;226:552-558. 